\title{
A Note on the Evaluation of the Complementary Error Function
}

\author{
By D. B. Hunter and T. Regan
}

Abstract. A modification is proposed to a method of Matta and Reichel for evaluating the complementary error function of a complex variable, so as to improve the numerical stability of the method in certain critical regions.

In the past twenty years, a number of methods have been proposed for evaluating the complementary error function

$$
\operatorname{erfc}(z)=\frac{2}{\sqrt{ } \pi} \int_{z}^{\infty} e^{-t^{\nu}} d t
$$

of a complex variable $z=x+i y$ by applying the trapezoidal rule or the mid-ordinate rule to the integral representation

$$
\operatorname{erfc}(z)=\frac{z e^{-z^{2}}}{\pi} \int_{-\infty}^{\infty} \frac{e^{-t^{2}} d t}{z^{2}+t^{2}} \quad(x>0)
$$

(see, e.g., Fettis [2], Luke [4], Hunter [3]). More recently, Chiarella and Reichel [1] have suggested a modification which greatly increases the accuracy of the approximation when $x$ is small. Their method has been further extended by Matta and Reichel [5].

The formula of Matta and Reichel [5] may be expressed in the form

$$
\operatorname{erfc}(z)=\frac{h z e^{-s^{2}}}{\pi} \sum_{r=-\infty}^{\infty} \frac{e^{-r^{2} h^{2}}}{z^{2}+r^{2} h^{2}}-R(h)-E(h) ;
$$

the summation represents the trapezoidal rule with interval $h>0$,

$$
\begin{aligned}
R(h) & =2 /\left(e^{2 \pi z / h}-1\right) & & \text { if } x<\pi / h, \\
& =1 /\left(e^{2 \pi z / h}-1\right) & & \text { if } x=\pi / h, \\
& =0 & & \text { if } x>\pi / h,
\end{aligned}
$$

and the error $E(h)$ is given by the expression

$$
E(h)=\frac{2 z e^{-s^{2}-2 \pi^{2} / h}}{\pi} \int_{-\infty}^{\infty} \frac{e^{-(t+i \pi / h) \cdot+2 \pi i t / h} d t}{\left[1-e^{-2 \pi^{2} / h^{2}+2 \pi i t / h}\right]\left[z^{2}+(t+i \pi / h)^{2}\right]}
$$

(the Cauchy principal value of the integral being taken if $x=\pi / h$ ). By using the fact that $\left|z^{2}+(t+i \pi / h)^{2}\right| \geqq\left|x^{2}-\pi^{2} / h^{2}\right|$, we may derive from (5) the inequality

Received October 4, 1971.

AMS 1969 subject classifications. Primary 3317; Secondary 6525.

Key words and phrases. Complementary error function, numerical integration, trapezoidal rule, mid-ordinate rule. 


$$
|E(h)| \leqq \frac{2\left|z e^{-z^{2}}\right| e^{-\pi^{2} / h^{2}}}{\pi^{1 / 2}\left(1-e^{-2 \pi^{2} / h^{2}}\right)\left|\left(x^{2}-\pi^{2} / h^{2}\right)\right|} \quad(x \neq \pi / h) .
$$

This inequality indicates that, even with relatively large values of $h$, the approximation obtained by omitting the error term $E(h)$ in (3) has considerable accuracy for nearly all values of $z$ in the right half-plane. In fact, Matta and Reichel [5] show that the accuracy is generally good even when $x=0$, despite the fact that the representation (2) then breaks down. However, the method obviously fails if $z=\operatorname{nih}$ ( $n$ an integer), and is numerically unstable if $z$ is close to one of those values.

Matta and Reichel [5] suggest that this difficulty may be overcome by merely altering the value of $h$-this will, of course, be ineffectual if $z$ is close to zero. The object of this note is to propose an alternative way round the difficulty. Instead of (3), we may use the formula

$$
\operatorname{erfc}(z)=\frac{h z e^{-z^{2}}}{\pi} \sum_{r=-\infty}^{\infty} \frac{e^{-(r+1 / 2)^{2} h^{2}}}{z^{2}+\left(r+\frac{1}{2}\right)^{2} h^{2}}+R^{\prime}(h)+E^{\prime}(h) ;
$$

the summation now represents the mid-ordinate rule, with interval $h$,

$$
\begin{aligned}
R^{\prime}(h) & =2 /\left(e^{2 \pi z / h}+1\right) & & \text { if } x<\pi / h, \\
& =1 /\left(e^{2 \pi z / h}+1\right) & & \text { if } x=\pi / h, \\
& =0 & & \text { if } x>\pi / h,
\end{aligned}
$$

and

$$
E^{\prime}(h)=\frac{2 z e^{-z^{2}-2 \pi^{2} / h^{2}}}{\pi} \int_{-\infty}^{\infty} \frac{e^{-(t+i \pi / h) 2+2 \pi i t / h} d t}{\left(1+e^{-2 \pi^{2} / h^{2}+2 \pi i t / h}\right)\left[z^{2}+(t+i \pi / h)^{2}\right]} .
$$

Inequality (6), with $E(h)$ replaced by $E^{\prime}(h)$, still holds.

Like the original method, this method breaks down for certain values of $z$, but, fortunately, not the same values as before-in fact, it fails if $z=\left(n+\frac{1}{2}\right)$ ih $(n$ an integer). This suggests that we adopt the following criterion:

(a) if $\frac{1}{4} \leqq \varphi(y / h) \leqq \frac{3}{4}$, use the formula given by (3);

(b) otherwise, use the formula given by (7).

Here, $\varphi(y / h)$ denotes the fractional part of $y / h$, i.e., $\varphi(y / h)=y / h-[y / h]$. Note that, in particular, (7) should be used if $z$ is real and small. For example, when $z=0$, Eq. (7), with $E^{\prime}(h)$ omitted, gives the value erfc(0) $=1$ exactly, whereas the first two terms on the right in (3) both become infinite.

The above criterion is important only if $z$ is close to one of the values $\frac{1}{2} n i h$, but it may safely be applied for any value of $z$ in the right half-plane. Finally, if $x<0$, we have

$$
\operatorname{erfc}(z)=2-\operatorname{erfc}(-z)
$$

Department of Mathematics University of Bradford

Yorkshire, England 
1. C. Chiarella \& A. Reichel, "On the evaluation of integrals related to the error function," Math. Comp., v. 22, 1968, pp. 137-143. MR 36 \#6117.

2. H. E. FETTIS, "Numerical calculation of certain definite integrals by Poisson's summation formula," $M T A C$, v. 9, 1955, pp. 85-92. MR 17, 302.

3. D. B. HunTER, "The evaluation of a class of functions defined by an integral," Math. Comp., v. 22, 1968, pp. 440-444.

4.' Y. L. LUKE, "Simple formulas for the evaluation of some higher transcendental functions," J. Mathematical Phys., v. 34, 1956, pp. 298-307. MR 17, 1138.

5. F. Matta \& A. Reichel, "Uniform computation of the error function and other related functions," Math. Comp., v. 25, 1971, pp. 339-344. 